\title{
Analysis of Fundamental Photodetection Noises and Evaluation of PIN and APD Photodiodes Performances using an Optical High Debit Transmission Chain Simulated by Optisystem
}

\author{
Abdelhakim Boudkhil \\ Department of Electronics, \\ Faculty of Technology, \\ Djillali Liabes University, \\ Sidi Belabbes, Algeria
}

\author{
Asmaâ Ouzzani \\ Department of Electronics, \\ Faculty of Technology, \\ Tahar Moulay University, \\ Saida, Algeria
}

\author{
Belabbes Soudini \\ Department of Electronics, \\ Faculty of Technology, \\ Djillali Liabes University, \\ Sidi Belabbes, Algeria
}

\begin{abstract}
Regarded as an extremity component for an optical communication chain, the photodetector plays a very significant role to detect and convert the optical ray penetrated by the optical fiber into an electric signal easier to exploit in the extraction of the information. Indeed, the noise of photodetection constitutes a precious phenomenon having several physical sources that affects the delivered signal at the output of the photodetector, focusing fundamentally on thermal noise "Johnson noise", quantum noise "Shot noise" and noise of multiplication "Multiplicative noise". This article aims to present a total study about the physical origins of fundamental photodetection noises by analyzing their behavior in PIN and APD photodiodes through an optical high debit transmission chain simulated by Optisystem software. The aim of this research extends also to evaluate and compare between $P I N$ and $A P D$ photodiodes in function of their functional parameters in terms of performance by measurement of signal-to-noise ratio in order to characterize these photodiodes.
\end{abstract}

\section{Keywords}

Optical communication, simulation, photodetector, semiconductor, PIN photodiode, APD photodiode, Optisystem.

\section{INTRODUCTION}

Today the new world of telecommunications represents a colossal circle of information where the evolution is incredibly high thanks to the actual communication systems which knew an effective extension of service diversification [1]. The application of optoelectronics in such systems involves optical methods which permit to convey and treat luminous signals using pure optical means; this manner presents numerous advantages comparing with the possibilities offered by electronics, for the simple reason that it is relatively easy to propagate luminous pulses with a very short duration of scale of the picoseconds, this property is attractive to realize more rapid and reliable systems "alloptics" involving radical changes in telecommunications industry [2].

Considered as a fundamental component in the reception module of the optical communication chain, the photodiode (PIN "Photodiode Intrinsic Negative" or APD "Avalanche PhotoDiode") plays a very important role in detecting luminous wave carrying information along the optical fiber, then transforms it into an electrical signal which will be easily exploited in the extraction of the transmitted information. In this purpose, several studies were being developed aiming to examine the major limitations caused in such optoelectronic component "the photodiode" by processing their physical origins.

In this context, B. Orsal and R. Alabedra [3] studied the photodiode characteristics by the measurements of $1 / f$ noise (Flicker noise which belongs to the excess noises). Using the $2^{\text {nd }}$ optical window $(\lambda=1300 \mathrm{~nm})$, they found that the Flicker noise is due to the dark current for two generations of photodiodes of different contact technology; this noise analysis showed that the quality of contacts of the $2^{\text {nd }}$ generation devices is better than the first generation. On the other hand, they analyzed [4] too the ionization by impact process in the APD photodiode (avalanche effect process) which has a relationship with noise of the multiplication introduced by holes which are the less ionizing carriers.

Otherwise, U. Ibrahim [5] evaluated and compared between the PIN photodiode and APD photodiode in terms of performance in an optical multiplexed transmission link using WDM "Wavelength Division Multiplexing" technique, he demonstrated that the capacity to transport data along 15 $\mathrm{km}$ can attain up to $5 \mathrm{Gbit} / \mathrm{s}$ per user if the $A P D$ photodiode is used in the receiver module.

P. Gopal [6] compared also between $P I N$ and $A P D$ performances in atmospheric turbulences of a satellite system for different modulation positions, he found that the performance of the $A P D$ photodiode is better than that of the PIN photodiode.

In the view of this importance, we are interested in the analysis of fundamental photodetection noises [7] generated in PIN and APD photodiodes consulted in an optical high debit transmission chain simulated by the optical software "Optisystem" [8].

This chain is considered as an environment for tests and observations where the objective is to evaluate PIN and $A P D$ photodiodes performances in terms of their functional parameters to practically compare between these two different photodiode types used as detectors for the actual optical communication systems. In fact, this work presents mainly:

- $\quad$ First, a description of photodetection principle created in semiconductors by explaining the optical-electrical interaction which is characterized by the "photons-electrons" interface. 
- $\quad$ Second, a specific view about $P I N$ and $A P D$ photodiodes which are responsible for photons-electrons conversion by presenting their structure, principle and functional parameters.

- Third, a deep study about fundamental photodetection noises summarized in Johnson noise, Shot noise and noise of multiplication by exposing the different physical sources that involve such phenomena in PIN and $A P D$ photodiodes.

- Fourth, interpretation and explanation of simulation results implemented by the Optisystem software through the optical high debit communication system chosen to analyze the photodetection noises and evaluate the PIN and $A P D$ photodiodes performances in function of signal-tonoise ratio $(S N R)$ in order to provide new perspectives for the future transmission axes.

\section{SEMICONDUCTORS AND PRINCIPLE OF PHOTODETECTION}

A semiconductor is a continuous crystalline environment characterized by its energetic band structure [9]: this energetic structure comprises a valence band which corresponds to the binder molecular states and a conduction band which includes the anti-binder molecular states, these two bands are separated by an energy range situated between the top of the valence band and the bottom of the conduction band known by band gap or energy gap.

The photodetection constitutes the core of optical communication systems because in an optical transmission system, the photoelectrical reception interface is the responsible for optimally detecting the luminous signal emitted by the optical source, reflected and guided by the optical fiber walls. This role is ensured by the photodiode which acts as a photon counter and a current generator.

The photodetection in semiconductors [7,9] operates on the creation of electron-hole pairs under the action of light: when a semiconductor is illuminated by a photon of an energy higher than or equal to its gap $\left(h . v \geq E_{g}\right)$, the absorbed photon carries the electron from the valence band to the conduction band (excited state), where it will be free to move in the crystalline network under the influence of an electric field, and the two generated carriers $(P$ and $N)$ are collected in a form of a photocurrent $I_{p h}$ (the number of electron-hole pairs is equal to the number of absorbed photons). The photodiode converts directly the incident photons into electrons which can be either ejected or liberated thanks to the photosensitive semiconductor properties that make the photodiode very fast in terms of photoelectrical conversion. This has practically developed the optical transmission systems for the very long distances thanks to the use of PIN and APD photodiodes. Fig 1.

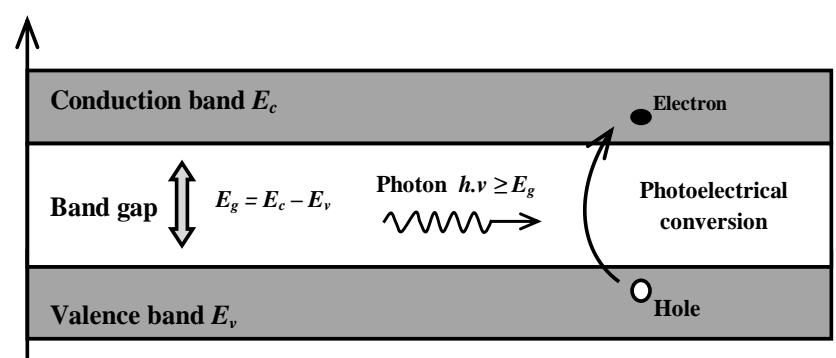

Fig 1: Semiconductor structure and photodetection principle "optical-electrical interaction"

\section{PIN AND APD PHOTODIODES}

The photodiode is a semiconductor photodetector that possesses two electrodes and a symmetric electrical characteristic [10]. It is used in optical transmissions for its high rapidity and responsivity, indeed, the most important structures disposed to convert the optical flow to an electrical current are:

\subsection{PIN photodiode}

The PIN photodiode [11] is constituted of three zones: the first is $P$-doped in order to create a hole excess, the second presents an intrinsic region " $i$ " called absorption zone and the third is $N$-doped in order to create an electron excess, from this the component name is formed. The width of the intrinsic zone is optimized in a way that maximizes PIN performances, so, it is preferred that this region be large from which the interest of $P I N$ photodiode.

In this context, in order to increase the photodiode responsivity, an anti-reflection dielectric coating is added to the photodetector device by consequence the majority of photons will be absorbed in the intrinsic zone. The photodiode response must be also very fast, it is limited by two factors: junction capacitance and transit time, so, it can be improved by increasing the reverse bias voltage which reduces these two factors [10]. Fig 2.

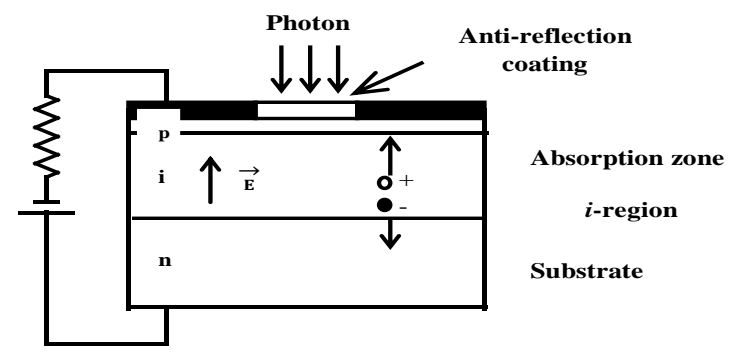

Fig 2: PIN photodiode structure

\subsection{APD photodiode}

In the $A P D$ photodiode, the created electrons are multiplied by the avalanche effect (multiplication process) in the intrinsic zone " $i$ " in order to obtain an important signal-tonoise ratio, the idea of using this internal multiplication phenomenon is applied so that the incident photon can generate several photoelectrons; this is to increase the electrical signal power which corresponds to the incident optical power. In fact, the $A P D$ photodiode is just a PIN photodiode conceived from a physical ionization by impact process in order to gain a multiplication effect. As a disadvantage, the response time of $A P D$ photodiode becomes longer due to the additional time occurred by the ionization phenomenon $[11,12]$. Fig 3.

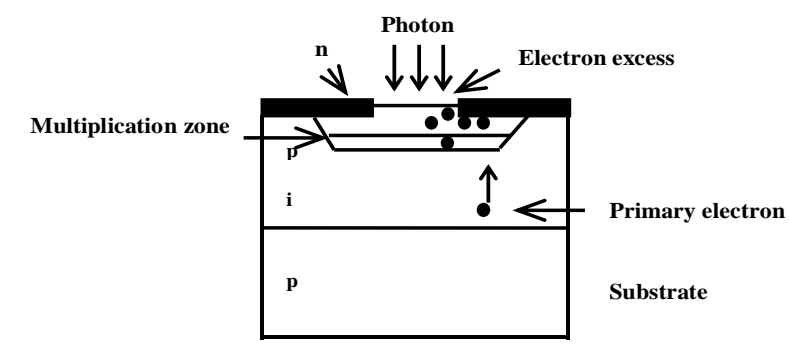

The $A P D$ photodiode is a refinement of the $P I N$ photodiode

Fig 3: APD photodiode structure 
$P I N$ and $A P D$ photodiodes are characterized by numerous functional parameters, among the main parameters which define such photodetectors there are [7]:

- Quantum efficiency “ $\boldsymbol{\eta}$ ”: It is a very important parameter which has relationship with the probability of creating electron-hole pairs through absorbed photons. It is defined as the fraction of incident photons (absorbed photons) which contribute to the external photocurrent. It can't be higher than 1, and it increases with the thickness of the absorption zone.

$$
\eta=\frac{I_{p h} / e}{P_{o p t} / h \cdot v}
$$

$\left(I_{p h}\right.$ : Photocourant, $P_{o p t}$ : Incident optical power, $e$ : Elementary electron charge, $h$ : Planck's constant $6.62610^{-34}$ J.s, $v$ : Luminous wave speed).

- Absorption coefficient “ $\alpha$ ”: One of the most important elements that determine the photodiode performances is the semiconductor absorption which is characterized by its absorption coefficient $\alpha$ which depends on the luminous wavelength $\lambda$ and of the energy gap $E_{g}$. If the photon energy h.v is higher than the semiconductor gap, there will be absorption, it is necessary then that the luminous wavelength $\lambda$ be less than the cut-off wavelength $\lambda_{c}$ $[13,14]$.

$$
d P_{a b s}(z)=P_{o p t} e^{-\alpha(\lambda) \cdot z} d z
$$

$\left(P_{a b s}:\right.$ Absorbed power, $\alpha$ : Absorption coefficient, $\lambda$ : Luminous wavelength, $z$ : Semiconductor depth)

Responsivity " $\boldsymbol{R}$ ": It is a very important parameter which is defined by the ratio of the photocurrent $I_{p h}$ on the received energy flux of photons (incident optical power) [13, 14].

$$
R(\lambda)=\frac{I_{p h}}{P_{o p t}}=\eta \frac{\lambda}{1,24} A / W
$$

- Noise Equivalent Power "NEP": The noise equivalent power is defined by the incident optical power for which the signal-to-noise ratio is equal to $1(S N R=1)$, in other words, the photocurrent $I_{p h}$ is equal to the noise current $I_{N}$. This parameter is inversely related with the photodiode directivity.

$$
N E P=\frac{I_{N}}{I{ }_{p h} / P_{o p t}}=D^{-1}
$$

- Gain “G”: It depends on the number of detected carriers in relation with photogenerated electron-hole pairs.

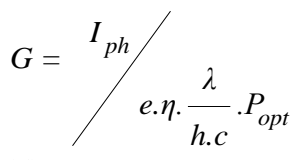

- Flux " $\Phi$ ": It is defined by the number of photons reaching into the active photodiode surface at a given wavelength per a unit of time.

$$
\Phi=P_{\text {opt }} \cdot \frac{\lambda}{h . c}
$$

\section{NOISE OF PHOTODETECTION}

At the reception, because of the corpuscular nature of electromagnetic luminous rays, absorption process of photons will be discrete and carries creation will be contingent in terms of time providing a random process, by consequence, the detection of incident optical rays becomes very inhibited because of photodetection noise. In this sense, the noise of photodetection [7] is generated in form of random intrinsic fluctuations which perturb the useful signal carrying information that makes them extraction very difficult at the photodetector output. In fact, the measurement of the photodetection noise in the presence of luminous signals is based on the evaluation of signal-to-noise that expresses the noise associated to the photocurrent generated through the conversion of photons into electron-hole pairs.

$$
S N R=\left(\frac{S}{B}\right)_{d B}=10 \log \frac{P_{o p t}}{N E P}
$$

There are five types of photodetection noise classified into two categories:

- Fundamentals noises: First, thermal noise "Johnson noise" which is due to the random collisions of carriers with crystal atoms which permanently vibrate that introduces a thermal agitation. Second, photonic or quantum noise "Shot noise" which is due to the collisions of incident photons. Third, noise of multiplication "Multiplicative noise" which is essentially a quantum noise amplified by the avalanche effect (multiplication process).

- $\quad$ Excess noises: First, noise with $1 / f$ called also "Flicker noise" which is associated to the presence of potential barriers in the electrical contacts. Second, noise of generation-recombination "G.R. noise" which is due to the random generation and recombination of carries because of gap traps.

In this context, the objective of this research is especially focused on the study of fundamental noises caused in the photodiode:

\subsection{Thermal noise "Johnson noise"}

This noise is the result of random carriers fluctuations caused in the photodiode resistance providing a thermal agitation, because the Brownien electron movement produces a random voltage at the resistance extremities.

$$
S_{i}(f)=\frac{4 \cdot k_{B} \cdot T}{R}
$$

( $k_{B}$ : Boltzmann's constant $1.38110^{-23} \mathrm{~J} / \mathrm{K}, T$ : Temperature in Kelvin, $R$ : Photodiode resistance)

The spectral density of current is independent of the frequency because it is the case of a white noise. In the 
photodiode, shunt resistance and series resistance are the two elements that represent the source of the thermal noise.

\subsection{Quantum noise "Shot noise"}

Shot noise or called Schottky noise depends on the corpuscular nature of electrons and their random emission and collection in terms of time:

$$
S_{i}(f)=2 . e . I
$$

It is also a white noise where the spectral density of current is uniform. It is due to the discrete fluctuations caused by the current containing electrons which are randomly distributed. In reality, this noise is caused by the dark current of the photodiode $I_{D}$ and the photocurrent $I_{p h}$ which are generated from the detectable luminous signal (absorbed photons).

$$
S_{i}(f)=2 \cdot e \cdot\left[I_{D}+I_{p h}\right]
$$

In the actual components, the noise is essentially due to the photocurrent $I_{p h}$ :

$$
I_{D} \ll I_{p h} \Rightarrow S_{i}(f)=2 . e . I_{p h}
$$

\subsection{Noise of multiplication "Multiplicative noise"}

The noise of multiplication is specifically generated in $A P D$ photodiode possessing an internal gain based on the multiplication method by impact of carriers in depletion region (junction region or space charge region). It is just a quantum noise caused in PIN photodiode which will be amplified in $A P D$ photodiode by the avalanche effect:

$$
S_{i}(f)=2 \cdot e \cdot I_{p h o} \cdot \bar{M}^{2}
$$

( $\bar{M}$ : Average value of multiplication factor of $A P D$ photodiode, $I_{p h o}$ : Primary photocourant)

\section{SIMULATION RESULTS}

The simulation part was executed using the Optisystem software which constitutes an interactive environment that permits to simulate, observe and analyze the transmitted signal at the level of all modules of the optical high debit transmission chain [8] considered as a model for the study in a form of schematic blocks as displays the figure 4, basing on the following parameters:

- $\quad$ Emitted power $P_{e}=50 \mathrm{~mW}$, binary debit $D=10$ Gbit/s, laser diode wavelength $\lambda=1552.52 \mathrm{~nm}$ corresponding to the $3^{\text {rd }}$ optical window, mono-mode fiber length $L_{F i b}=50$ $\mathrm{Km}$, photodiode sensitivity $S=0.8 \mathrm{~A} / \mathrm{W}$, photodiode dark current $I_{D}=5 \mathrm{nA}$

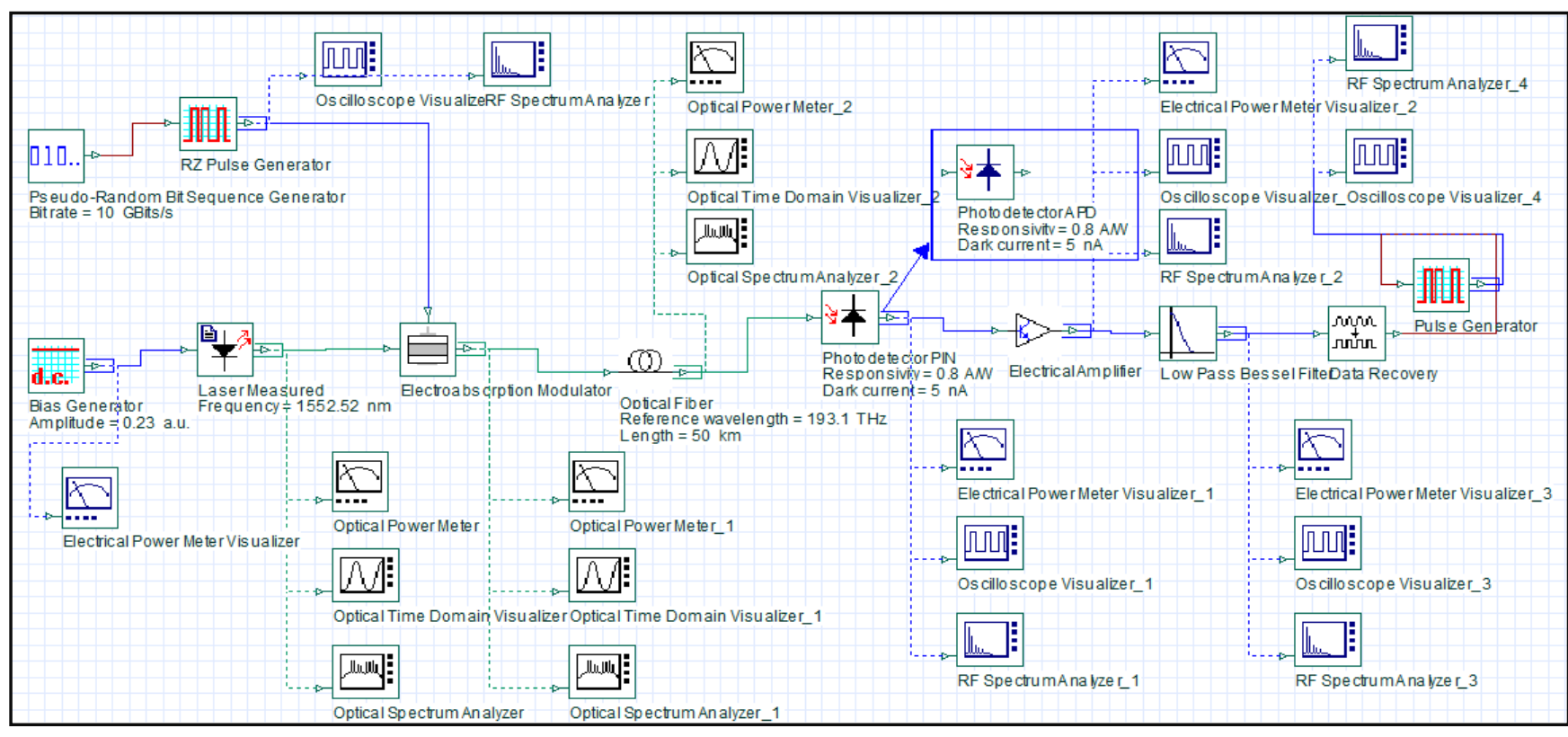

Fig 4: Model of simulation realized by Optisystem: Optical high debit transmission chain " $P_{e}=50 \mathrm{~mW}, D=10 \mathrm{Gbit} / \mathrm{s}$, $\lambda=1552.52 \mathrm{~nm}, L_{F i b}=50 \mathrm{Km}, S=0.8 \mathrm{~A} / \mathrm{W}, I_{D}=5 \mathrm{nA}, G_{A P D}=3 \mathrm{~dB} "$

The aim of this study is to examine the noise of photodetection caused in PIN and APD photodiodes used as detectors in the transmission chain basing on a temporal visualization (in terms of time using an optical time domain visualizer) or a spectral visualization (in terms of frequency using an optical spectrum analyzer).

In specific, it is distinguished that the photodiode constitutes the seat of the noise which is observed additive to the useful signal; this noise has a random character manifested by parasitic fluctuations that distort the electrical pulses containing information as shows the figure 5. It is the noise of photodetection [15] whose the sources are internal generated in the photodiode core; this noise has a low power but it equivocally influences the received signal consequently the transmitted information.

The objective of this study extends to define the dominant noise contribution in both PIN and APD photodiodes considered as detectors for the studied transmission chain in order to compare and evaluate their performances, in fact, this simulation consists of two major steps: 


\subsection{Analysis of photodetection noise in PIN and APD photodiode}

This first step consists to simulate the optical chain using respectively the PIN photodiode and the $A P D$ photodiode in order to visualize the shape of thermal and quantum noises caused in these photodiodes using a power spectrum analyzer.

As displays the figure $6(\mathrm{a}, \mathrm{b})$, the thermal noise power caused in the PIN photodiode is higher than it in the APD photodiode.

On the contrary, the quantum noise power caused in the $A P D$ photodiode is denser than it in the PIN photodiode as displays the figure $7(a, b)$.

The dominant noise in the PIN photodiode is that of thermal noise derived from the various circuits contained in the photodiode such as the resistance and the preamplifier which cause thermal agitation of electrons which consequently influences on the photodiode responsivity, however the $A P D$ photodiode resists against the thermal noise thanks to its internal gain produced by the avalanche process, but this process causes a rising of quantum noise that reduces the photodiode detection speed, this is due to the noise of multiplication generated particularly in $A P D$ photodiode by impact of carriers in the depletion region. In this view, the optical system was simulated again using an $A P D$ photodiode by varying its internal gain from 2 to $15 \mathrm{~dB}$. The figure 8 presents both the detected signal and the noise of photodetection measured by an electrical power measurer, and the signal-to-noise ratio calculated from the two previous values in function of the avalanche factor.

It is observed that more the internal $A P D$ gain increases more the detected signal and the photodetection noise increase, this involves a degradation of signal-to-noise ratio caused by the quantum noise which is amplified more rapidly than the detected signal. This phenomenon is due to the noise of multiplication also known as avalanche noise because it is only generated in $A P D$ photodiode [4]

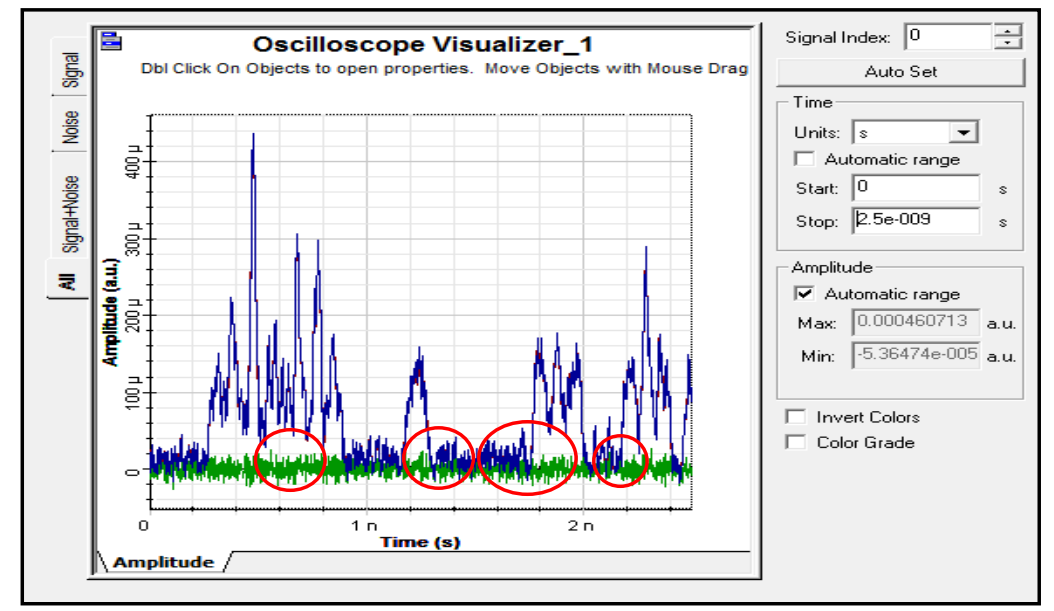

Fig 5: PIN photodiode output (detected signal in blue) - Noise of photodetection (noise signal in green) - Fluctuations (distorted signal surrounded by the red circles)

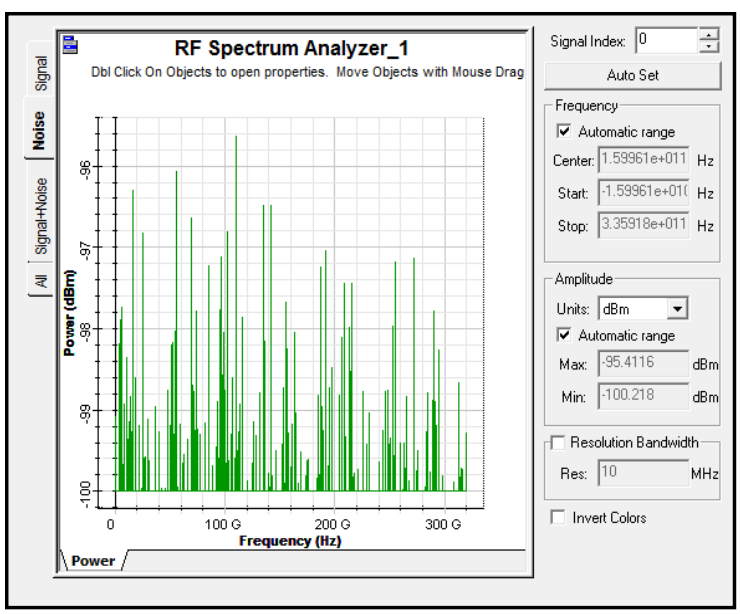

(a) PIN photodiode

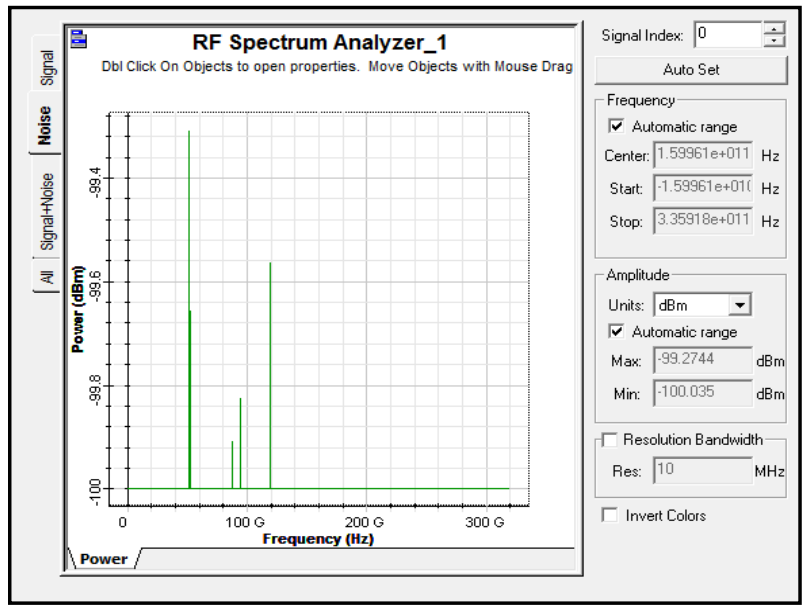

(b) $A P D$ photodiode

Fig 6: Spectral visualization of thermal noise “Johnson noise" caused in PIN and APD photodiodes 


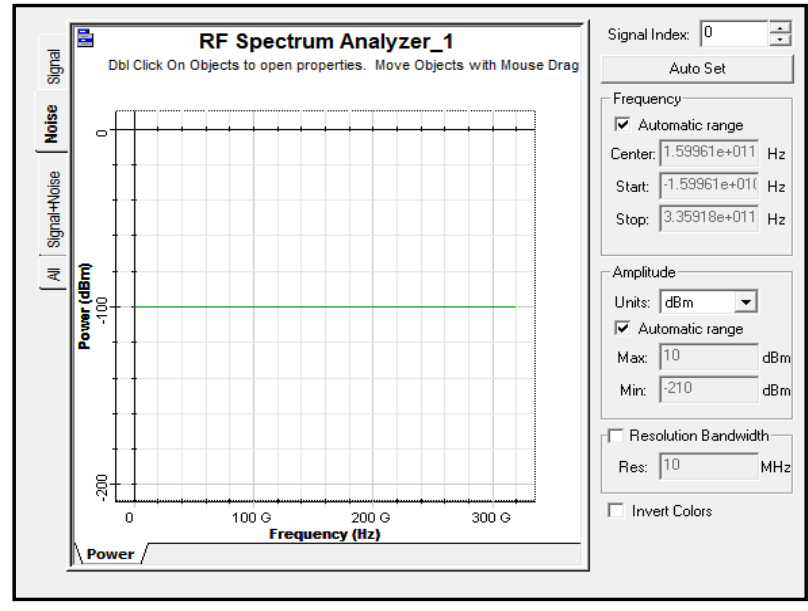

(a) PIN photodiode

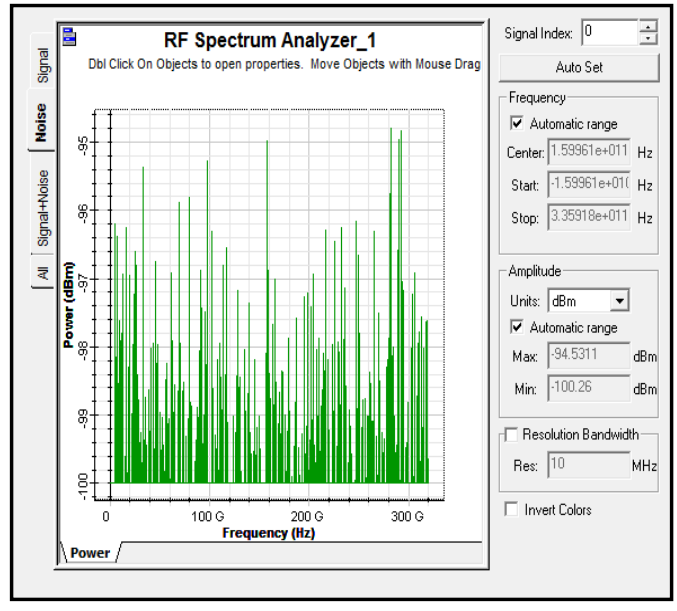

(b) $A P D$ photodiode

Fig 7: Spectral visualization of quantum noise "Shot noise" caused in PIN and APD photodiodes

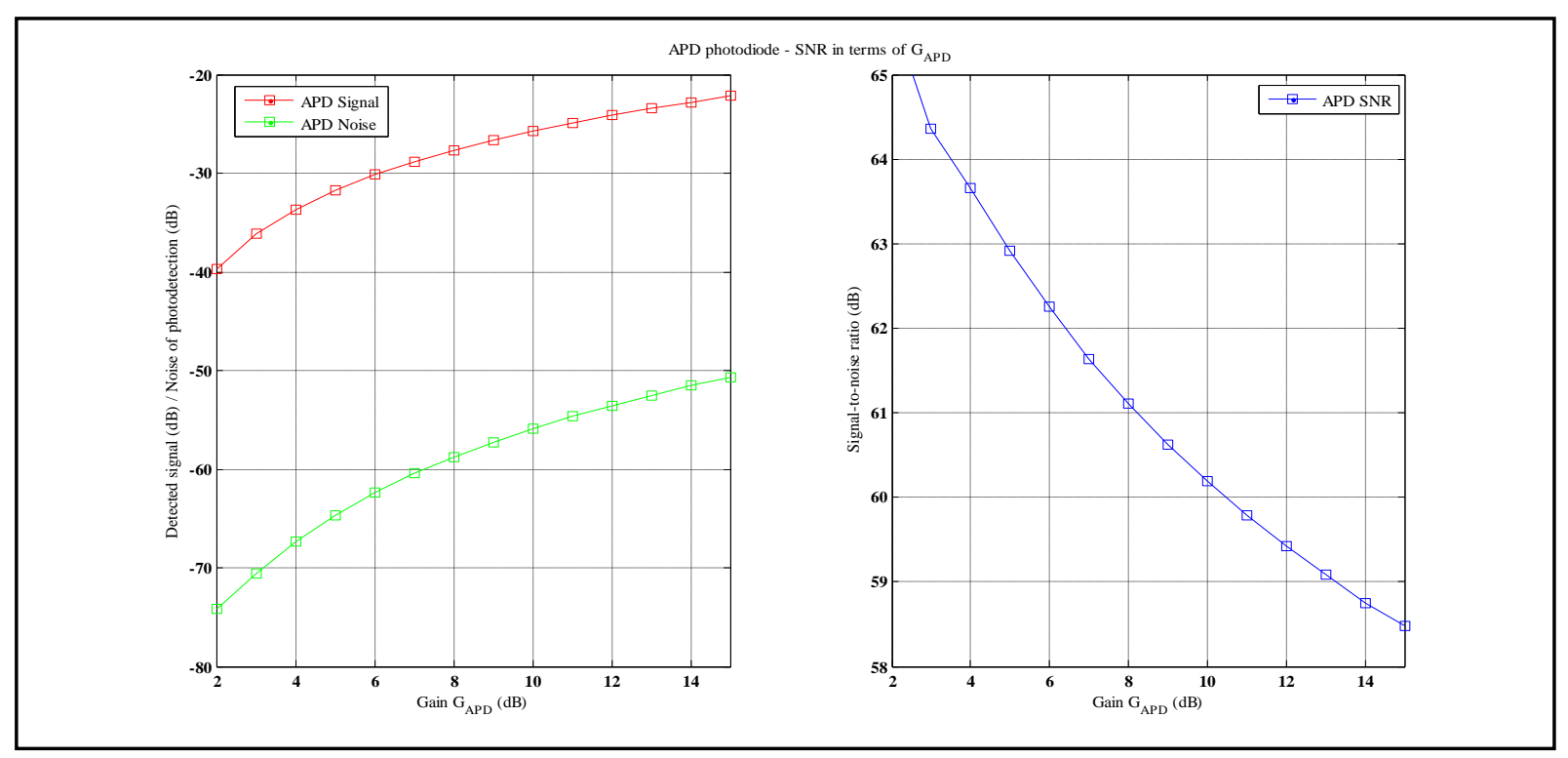

Fig 8: Signal-to-noise ratio in terms of internal gain of the avalanche photodiode $A P D-S N R=f\left(G_{A P D}\right)$

\subsection{Evaluation of performances of PIN and APD photodiodes}

The objective of this second step is to study the performance of $P I N$ and APD photodiodes according to the following functional parameters: emitted power $P_{e}$, debit $D$, responsivity $R$ and dark current $I_{D}$ by measurement of the detected signal issued at the photodiode output and the photodetection noise generated in the photodiode core by using the electrical power measurer and then calculate the signal-to-noise ratio in decibel [16].

In this step a binary debit of $2.5 \mathrm{Gbit} / \mathrm{s}$ was contributed with using for the first time a PIN photodiode and for the second time an $A P D$ photodiode by varying in every time only one of the previous parameters for each photodiode.

The results are represented in the following curves shown respectively in the figures $9,10,11,12$ :

- In terms of the emitted power $-S / B=f\left(P_{e}\right)$ : In the figure 9 , it is clearly observed that more the input power " $P \mathrm{e}$ " increases more the detected signal is better, however the noise is relatively constant and seems lower than the received signal. This expresses that the signal-to-noise ratio rises with the emitted power. Besides, the comparison between the PIN and $A P D$ photodiodes justifies that the $A P D$ photodiode is more advantageous than the PIN photodiode for the low powers, by contrast the PIN photodiode remains always more efficient for the high powers upper than $0.7 \mathrm{~W}$ [17].

Subsequently, the emitted power will be equal to $50 \mathrm{~mW}$, a value which belongs to the low powers range and justifies that the $A P D$ performances will be better than the PIN performances in terms of signal-to-noise ratio for the following sub-parts.

- In terms of debit $-\boldsymbol{S} / \boldsymbol{B}=\boldsymbol{f}(\boldsymbol{D})$ : The figure 10 shows that the input binary debit " $D$ " varies from $100 \mathrm{Mbit} / \mathrm{s}$ to 55 Gbit/s, it notes that more the input debit increases more the photodetection noise increases but the detected signal is relatively attenuated. In fact, raising the data debit reduces the signal-to-noise ratio that decreases therefore the transmission performances. Finally, if we need to keep a good performance for the studied system, it should not exceed a debit of $25 \mathrm{Gbit} / \mathrm{s}$, because above this value, the signal-to-noise ratio fall and becomes not acceptable.

- In terms of responsivity $-S / B=f(R)$ : In the figure 11 , the responsivity " $R$ " varies from 0.1 to $1 \mathrm{~A} / \mathrm{W}$, it is directly 
observed that more the responsivity increases more the detected signal increases, however the photodetection noise takes very low values that makes the signal-to-noise ratio better consequently the transmission performances. This demonstrates that the signal-to-noise ratio is in a good agreement with the responsivity of the photodiode, PIN or APD and becomes adequate above a value of $0.3 \mathrm{~A} / \mathrm{W}$.

- In terms of dark current $-S / B=f\left(I_{D}\right)$ : The figure 12 shows that the dark current " $I_{D}$ " varies from 0 to $10000 \mathrm{nA}$. It is simply observed that more the dark current increases and takes higher values, the detected signal, the noise of photodetection and the signal-to-noise ratio keep always constant values. This proves that the dark current is negligible for the photoconductor mode [7], its value is very low to be considered in the generation of the noise of photodetection that confirms what was already mentioned in the section $(4, b)$.

Finally, the $A P D$ photodiode retains always the better performance comparing with the PIN photodiode for the reason that the manipulation used particularly a low input power equal to $50 \mathrm{~mW}$.

\section{CONCLUSION}

This work has mainly aimed to analyze and describe the fundamental photodetection noises caused in PIN and APD photodiodes; this permitted to study the physical sources generating the noise of photodetection which displays internal and random, and noticeably affects the photodiode signal. This research has also aimed to characterize these photodetectors by examining their performances in terms of different functional parameters by measurement of the signal-to-noise ratio which constitutes a very important factor to determine the quality of the optical detection for the optical high debit communication chain chosen as a model of simulation and study; this led to conclude that these parameters constitute always major factors in evaluating $P I N$ and $A P D$ performances, making their choice more judicious for high transmission quality in the various optical communication systems having an important role in the new telecommunications world because today we can't speak about telecommunications without mention the optical communication systems characterized by a grand demand for their exceptional quality offering high debit, long distance of propagation and strong immunity against perturbations.

Finally, it is very interesting to extend this research by studying the other photodetection noises which are the excess noises
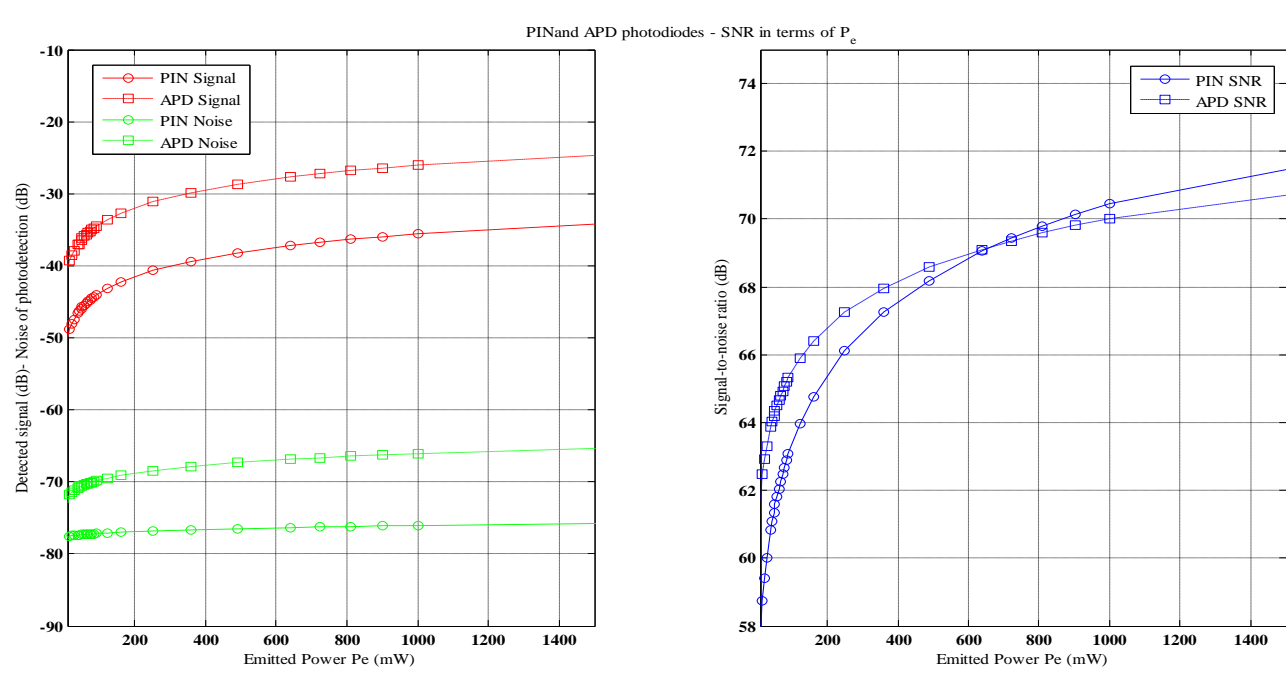

Fig 9: Signal-to-noise ratio in terms of emitted power for $P I N$ and $A P D$ photodiodes $-S N R=f\left(P_{e}\right)$
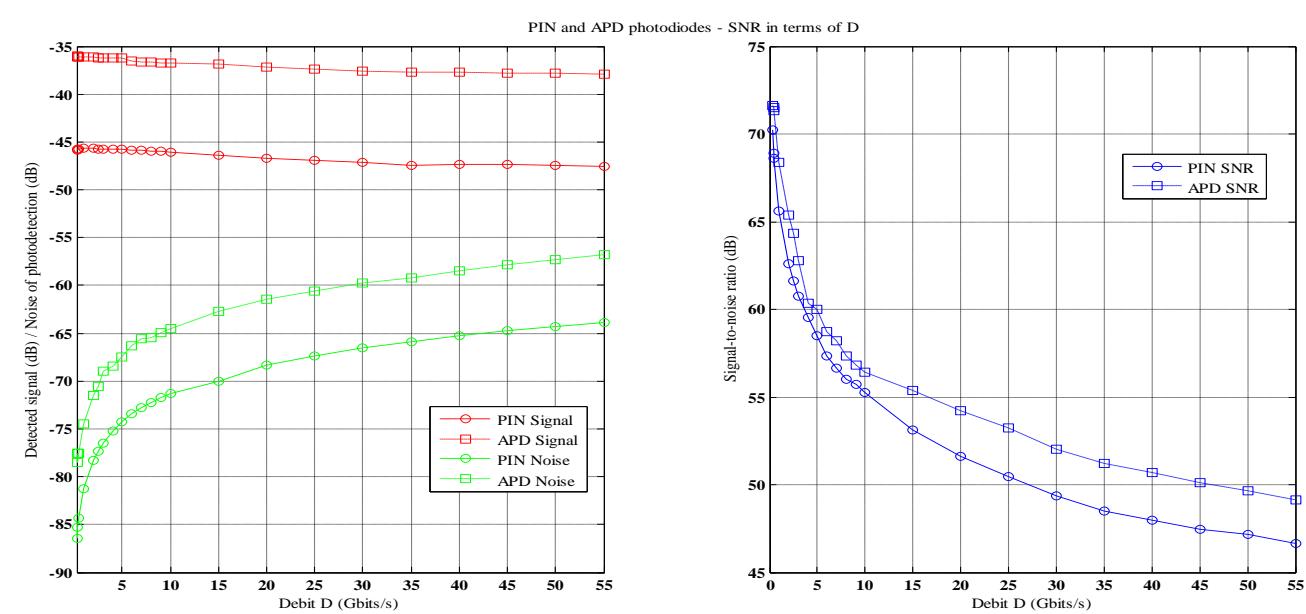

Fig 10: Signal-to-noise ratio in terms of binary debit for $P I N$ and $A P D$ photodiodes $-S N R=f(D)$ 


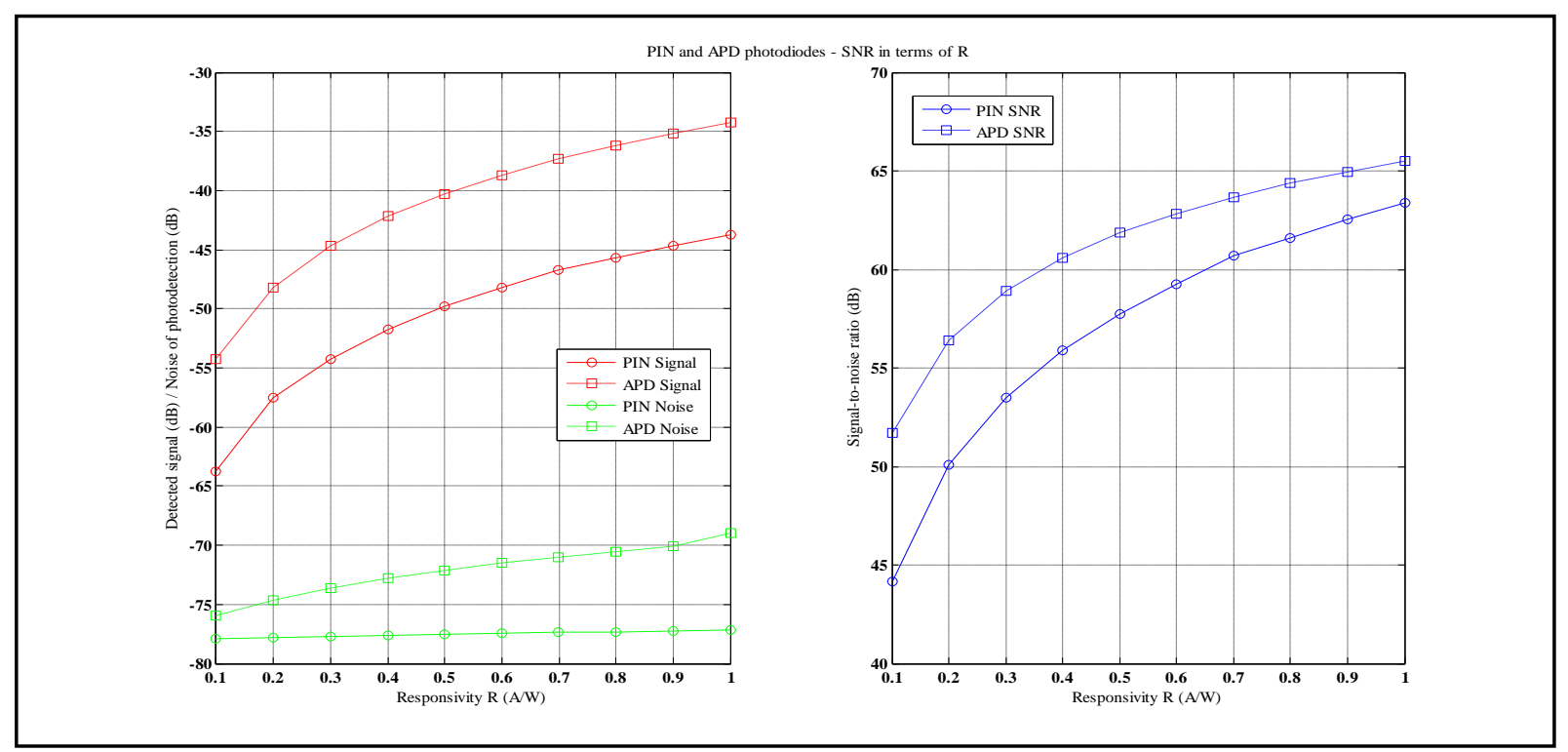

Fig 11: Signal-to-noise ratio in terms of responsivity for $P I N$ and $A P D$ photodiodes $-S N R=f(R)$

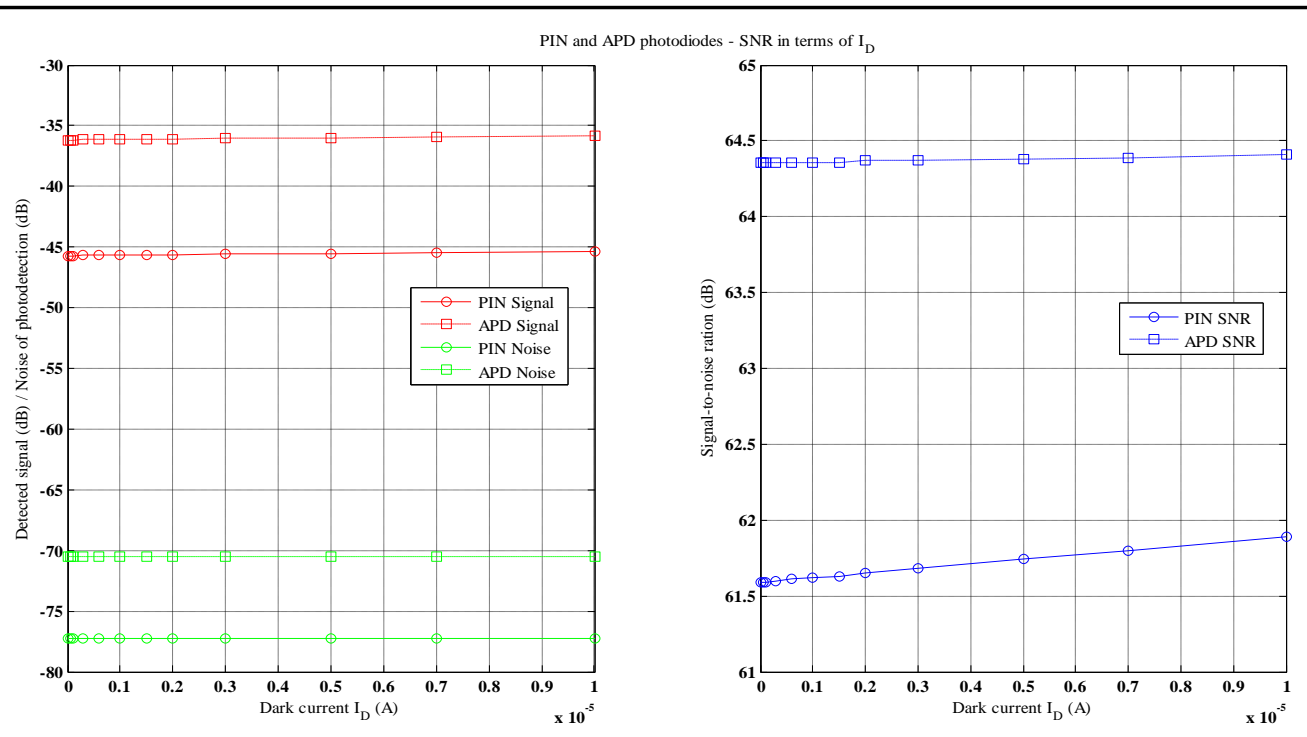

Fig 12: Signal-to-noise ratio in terms of dark current for $P I N$ and $A P D$ photodiodes $-S N R=f\left(I_{D}\right)$

summarized in "Flicker noise" and "generationrecombination noise", as it is very important to model all types of photodetection noise in order to get a good performance of photodetector that establishes a topical subject making the optical communication systems more efficient.

\section{REFERENCES}

[1] Servin, C. 2009. Networks and telecoms / courses and exercises. Dunod. Paris.

[2] Brahimi, H. 2010. Study of Microwave Optical System Noise. Modelization, Characterization and Application of Phase Metrology Noise and Frequency Generation. Doctoral thesis. Speciality of Micro-waves, Electromagnetism and Optoelectronics. University of Paul Sabatier Toulouse III.

[3] B. Orsal, R. Alabedra, C. Maille, C. Boisrobert, D. Morvan, J. Meslage and G. Pichard, "Characterization of PIN photodiodes noise. $\mathrm{Hg}_{1-\mathrm{x}} \mathrm{Cd}_{\mathrm{x}}$ Te with $\lambda=1,3 \mu \mathrm{m}$ ", Journal of Applied Physics, Vol 19, Issue 2, February 1984, 63-67.
[4] R. Alabedra, B. Orsal, A. H. Maatougui, K. D. Raniriharinosy, P. Gori and C. Boisrobert, "Report of ionization coefficients and noise in avalanche photodetectors $\mathrm{Hg}_{0,44} \mathrm{Cd}_{0,56} \mathrm{Te}$ with $\lambda=1600 \mathrm{~nm}$, Annals of Telecommunications, Vol 43, Issue 34, March/April 1988, 117-122.

[5] U. Ibrahim, H. Abbas, R. Faizan and S. F. Shaukat, "Performance evaluation of photoreceivers in WDM passive optical networks", $6^{\text {th }}$ IEEE International Conference on Engineering Technologies, Islamabad, October 2010.

[6] P. Gopal , V. K. Jain and S. Kar, "Performance comparison of PIN and APD based FSO satellite systems for various pulse modulation schemes in atmospheric turbulence", Communications and Network Journal, Scientific Research, September 2013.

[7] Decoster, D. and Harari, J. 2002. Optoelectronic detectors. Hermes-Science. Paris. 17-20, 26-28, 205-214. 
[8] A. Boudkhil, A. Ouzzani and B. Soudini, "Introduction to the optical communications by simulating an optical high debit transmission chain using Optisystem with comparison of optical windows", International Journal of Computer Networks and Communications Security, Vol 3, Issue 2, February 2015, 53-62.

[9] Voos, M. Drouhin, H. J. and Drevillon, B. 2001. Semiconductors and components. Physical Program, Technological Innovation. Electrical Engineering, Polytechnic Institute, Palaiseau. Paris. 7-44.

[10] D. Courjon, "Photodiode characteristics and applications", OSI Journal, Optoelectronics, 2009.

[11] Boisrobert, C. and Toffano, Z. 2001. Master and ASD optics and optoelectronics courses. University of Nantes.

[12] K. Hinton and T. Stephen, "Modeling high speed optical transmission systems", Selected Areas in Communication, IEEE Journal, Vol 11, Issue 3, April 1993, 380-392.
[13] E. Cassan, "Introduction to the optical telecommunications by simulating simple systems", Journal of Science Teaching, Information Technologies and Systems, J3EA, Vol 2, June 2003.

[14] Kasap, S. O. 2001. Optoelectronics and photonics Principles and practices. Prentice Hall Upper Saddle River, NJ.

[15] Boudkhil, A. Ouzzani, A. and Soudini, B. 2015. Optical communication - Noise of photodetection. European University Edition. Sarrebruck.

[16] A. Boudkhil, A. Ouzzani and B. Soudini, "Evaluation of PIN and APD photodiodes performances in an optical high debit transmission chain via Optisystem", Mediterranean Telecommunication Journal, Vol 5, Issue 1, March 2015, 1-6.

[17] Gouy, J. P. 1989. Comparative Study of the PIN Photodiode, APD Photodiode and of Photoconductors based on IIIV Materials. Doctoral thesis. Speciality of Electronics. University of Flandres Artois Lille. 